\title{
A!
}

This is an electronic reprint of the original article.

This reprint may differ from the original in pagination and typographic detail.

Puska, M.J.; Ogando, E.; Zabala, N.

\section{Shell and supershell structures of nanowires, a quantum-mechanical analysis}

Published in:

Physical Review B

DOI:

10.1103/PhysRevB.64.033401

Published: 06/06/2001

Document Version

Publisher's PDF, also known as Version of record

Please cite the original version:

Puska, M. J., Ogando, E., \& Zabala, N. (2001). Shell and supershell structures of nanowires, a quantummechanical analysis. Physical Review B, 64(3), 1-4. [033401]. https://doi.org/10.1103/PhysRevB.64.033401

This material is protected by copyright and other intellectual property rights, and duplication or sale of all or part of any of the repository collections is not permitted, except that material may be duplicated by you for your research use or educational purposes in electronic or print form. You must obtain permission for any other use. Electronic or print copies may not be offered, whether for sale or otherwise to anyone who is not an authorised user. 


\title{
Shell and supershell structures of nanowires: A quantum-mechanical analysis
}

\author{
M. J. Puska \\ Laboratory of Physics, Helsinki University of Technology, P.O. Box 1100, FIN-02015 HUT, Finland \\ E. Ogando \\ Elektrika eta Elektronika Saila, UPV-EHU 644 P.K., 48080 Bilbo, Spain \\ N. Zabala \\ Elektrika eta Elektronika Saila, UPV-EHU 644 P.K., 48080 Bilbo, Spain \\ and Donostia International Physics Center (DIPC) and Centro Mixto CSIC-UPV/EHU 1072 P.K., 20080 Donostia, Spain
}

(Received 16 June 2000; revised manuscript received 25 January 2001; published 6 June 2001)

\begin{abstract}
The stability of sodium nanowires is studied by modeling them as infinite uniform jellium cylinders and solving self-consistently for the electronic structure. The total energy per unit length oscillates as a function of the wire radius giving a shell structure. The amplitude of the energy oscillations attenuates regularly, reflecting a supershell structure. We compare our theoretical results with recent experiments [A. I. Yanson et al., Nature 400, 144 (1999); Phys. Rev. Lett. 84, 5832 (2000)] performed by the mechanically controllable break junctions (MCB) technique. The comparison clarifies the origin of the observed shell structure and especially the formation of the quantum beats of the supershell structure and supports the conclusions based on an earlier semiclassical model. The comparison is also a quantitative test for the reliability of the simple stabilizedjellium model as well as for the accuracy of the equation used to relate the conductivity and the area of the narrowest point of the constriction.
\end{abstract}

DOI: 10.1103/PhysRevB.64.033401

PACS number(s): 73.21.-b, 73.40.Jn

During the last ten years metallic nanowires have attracted a large amount of experimental and theoretical research. ${ }^{1}$ The motivation for these studies arises partly from the miniaturization of electronics components: when the feature sizes continuously decrease the quantum effects become more pronounced and their deep understanding becomes essential. On the other hand, the quantum effects themselves, such as the quantization of the electric conductance, have introduced a very rich research field within basic physics.

Yanson et al. ${ }^{2}$ used the mechanically controllable break junction (MCB) technique in studying the conductance of sodium wires. They recorded the quantized conductance values observed in $\sim 10^{4}$ breaking and rejoining contact events and formed histograms for the observed values. The conductance can be related by a semiclassical expression to the radius of the narrowest cross section of the nanowire ${ }^{3}$ and a histogram showing the occurrence frequencies of different radii is obtained. When the sample temperature is not too low, i.e., it is of the order of $80 \mathrm{~K}$, the histogram shows peaks periodically as a function of the radius. ${ }^{2}$ This indicates a kind of shell structure. A peak means that a nanowire with that particular radius of the narrowest cross section is especially stable. The finite temperature is explained to clear up the quantum shell structure because ions can rearrange to minimize the total energy. The smoothing of the surfaces by adatom diffusion is also expected to make the constriction more "ideal" for the shell structure. The shell structure of an electronically open nanowire is analogous with those found, e.g., for atomic clusters that are closed systems of electron states. ${ }^{4}$ Peaks up to the conductance value of about $120 G_{0}$, where $G_{0}=2 e^{2} / h$ is the quantum of conductance, have been observed. ${ }^{2}$ Recently, Yanson et al. ${ }^{5}$ have shown that for certain peaks the amplitude is diminished. These amplitude modulations or "quantum beats" correspond to the supershell structure of the nanowires.

The shell structure of sodium nanowires can be understood $^{2}$ by semiclassical arguments: ${ }^{6}$ The oscillation periods for the density of states at the Fermi level [DOS $\left.\left(E_{F}\right)\right]$ correspond to classical closed orbits in the minimum circular cross section so that Bohr-Sommerfeld quantization condition is fulfilled, i.e., the path length is an integer multiple of the Fermi wavelength. The dominating orbits give the shell structure that appears as regular oscillations of $\operatorname{DOS}\left(E_{F}\right)$ and other physical quantities as a function of the minimum radius. Furthermore, the difference of the path lengths between two dominating orbits gives the supershell structure. The free energy of a nanowire can be calculated ${ }^{2,5}$ using a corresponding semiclassical expansion. ${ }^{7,8}$ The oscillations in the free energy are reflected in the stability or in the abundance of nanowires with different radii.

In this Brief Report we report a fully quantum-mechanical analysis of the shell and supershell structures of sodium nanowires. We model the wires as infinitely long, constantradius stabilized-jellium cylinders defined by two parameters, the density parameter $r_{s}=3.93 a_{0}$ for the rigid positive background charge and the radius $R$. The electronic structure is solved self-consistently within the density-functional theory ${ }^{9}$ (DFT) using the local-density approximation (LDA) for the exchange-correlation effects. The Kohn-Sham equations are solved on a radial mesh. ${ }^{10} \mathrm{We}$ use the stabilizedjellium model because it describes many properties of simple metals well, even with a quantitative accuracy. It is also simple enough to enlighten only the essential physical features of the phenomena studied.

The DFT calculation gives the total energy of the electron-background charge system. We calculate the surface 


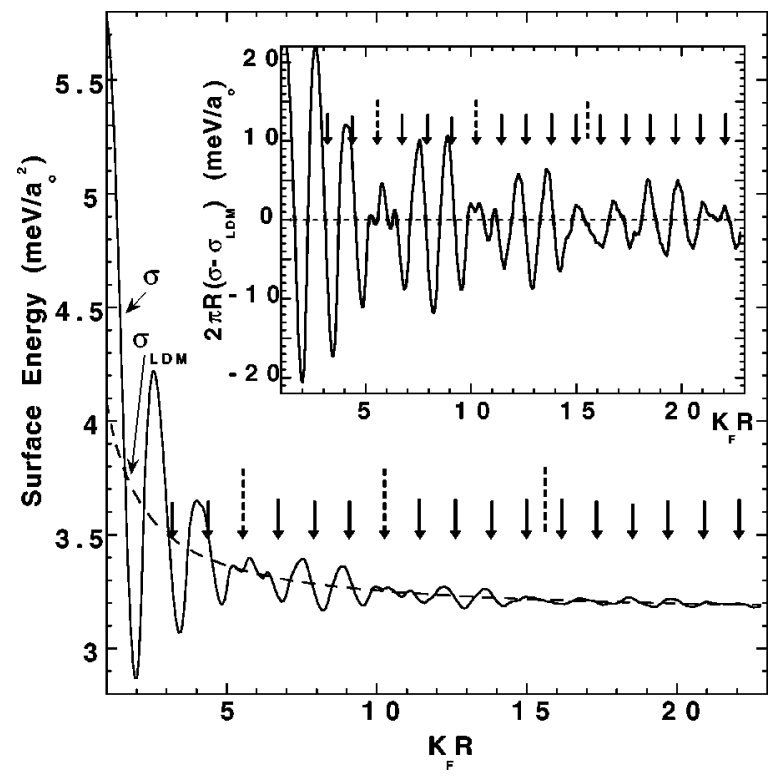

FIG. 1. Surface energy for Na stabilized-jellium wires. The result of the liquid-drop model is given as a dashed line. Arrows pointing downwards give the peak positions in the experimental conductance histograms (Ref. 2) converted to the wire radii by using Eq. (1) with $\alpha=-0.1$. The rescaled positions of the experimentally observed beats are given by vertical dashed lines. The inset shows the oscillating part of the total energy per unit length.

energy $\sigma$ and the energy per unit length of the wire $E_{\text {tot }} / L$. They are related as $\sigma=(1 / 2 \pi R)\left\{E_{\mathrm{tot}} / L-\pi R^{2} E_{\mathrm{bulk}}\right\}$, where $E_{\text {bulk }}$ is energy of the homogeneous bulk system per unit volume. Figure 1 shows the surface energy as a function of $K_{F} R$, where $K_{F}$ is the Fermi wave vector for sodium. It shows oscillations reflecting the radial confinement of electron states and the appearance of new occupied subbands below the Fermi energy when $R$ increases. At large $R, \sigma$ approaches the energy of the flat surface. The energy minima in Fig. 1 correspond to wires with enhanced stability, a kind of "magic radii" system. ${ }^{11}$ In Fig. 1 we show also the surface energy $\left(\sigma_{\mathrm{LDM}}\right)$ obtained using the so-called liquid-drop model. ${ }^{12,10}$ The parameters of the model are from independent calculations for systems of nonconfined electrons. ${ }^{12}$ The liquid-drop model therefore provides the smoothed energy ${ }^{6}$ that should be subtracted when studying the shell and supershell effects. This is what we have done in the inset of Fig. 1 giving the oscillating part of the total energy per unit length.

Our idea is that the stability of a wire is reflected in its abundance. The narrowest point determines the stability and also the conductance $(G)$. Conductance histograms should therefore give the relative stabilities of wires with different radii. Yanson $e t \mathrm{al}^{2,5}$ used the simple parabolic function proposed by Torres et al. ${ }^{3}$ to relate the conductance with the wire radius. The function is based on quantum-mechanical calculations for noninteracting electrons in a hard-wall cylindrical potential. Garcia-Martin et al. ${ }^{13}$ introduced a more general expression,

$$
G / G_{0}=\left(\frac{K_{F} R}{2}\right)^{2}\left(1-\alpha \frac{4}{K_{F} R}\right)
$$

in order to take the spillover of the electron density into account. They quoted the value of $\alpha \simeq 0.12$ corresponding to the experimental work function and Fermi energy of sodium. The value of $\alpha=0.5$ reproduces the hard-wall result and $\alpha$ $=0$ yields the classical Sharvin limit. ${ }^{14}$

In the quantum treatment, the conductance through a small contact is obtained from the Landauer multichannel formula ${ }^{15}$

$$
G / G_{0}=\sum_{m} \sum_{n} T_{m n}
$$

where $T_{m n}$ is the transmission probability of channel $m n$ and the sum runs over all occupied subbands. In the cylindrical symmetry the channels are given by the quantum numbers $m$ and $n$, corresponding to the angular momentum and the nodes of the radial wave function, respectively. Neglecting electron reflection effects, $T_{m n} \approx 1$, and the conductance is simply given by the number of subbands under the Fermi level (modes with $m \neq 0$ are doubly degenerate). We have used this approach with our stabilized-jellium model results, obtaining a stepped conductance as a function of $K_{F} R$. A fit with Eq. (1) gives $\alpha=-0.1$. The low value reflects the soft potential in the self-consistent jellium model. However, in the conductance-radius correspondence there is an uncertainty due to the conductance plateau width, which is of the order of $K_{F} R=1$ or even larger.

The arrows pointing downwards in Fig. 1 denote the peak (shell) positions in the experimental data by Yanson et al., ${ }^{2}$ rescaled to correspond to the stabilized-jellium background radii. The dashed vertical lines give the positions of reduced amplitude (beats) according to the data by Yanson et al..$^{5}$ The first two beat positions coincide with the third and the seventh conductance peak positions, and the third beat is in the middle of the 11th and 12th peaks. The correlation between the peak positions and the minima of the surface energy is quite good. The distances between adjacent peaks and between adjacent energy minima are similar but the minima are shifted slightly to higher $K_{F} R$ values. At about $K_{F} R=6$ and 10.5 there are no clear minima because the surface energy oscillates moderately around the liquid-drop line. But these positions correspond to the beat regions of the supershell structure in agreement with experiment. ${ }^{5}$ In the relatively wide region of $K_{F} R \approx 14, \ldots, 17$ the oscillations in the surface energy have a smaller amplitude and they are somewhat "messy." Here the correlation between the measured peak positions and the energy minima is not good. This region corresponds to the third observed beat. ${ }^{5}$ Above the beat region, at about $K_{F} R \approx 17, \ldots, 20$ the oscillations become "cleaner" again before the next beat region at around $K_{F} R$ $\approx 21$. The reason for the shifts between the measured peak positions and calculated energy minima may be the impurity scattering. It reduces the conductance, especially in the case of higher conductance values. ${ }^{16}$ Therefore the positions of the observed peaks in Fig. 1 should be at higher $K_{F} R$ values, especially in the region of higher $K_{F} R$. This would improve the agreement between theory and experiment.

Recently Kassubek et al. ${ }^{17}$ studied the stability of uniform cylindrical nanowires against perturbations in the radius 


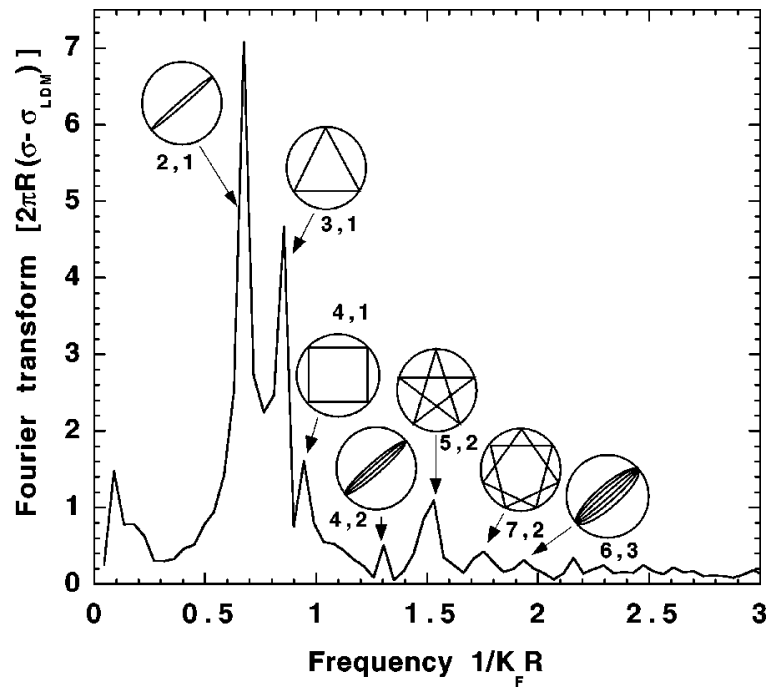

FIG. 2. Fourier transform of the oscillating part of the total energy per unit length for Na stabilized-jellium wires (the inset of Fig. 1). The tips of the arrows give the frequencies [Eq. (3)] for different classical orbits inside a circle.

along the wire axis. They calculated the grand canonical potential of the system for the temperature of $80 \mathrm{~K}$ using a semiclassical approach for the density of electron states. They found regions of stability as a function of the wire radius and constructed a conductance histogram assuming that the probability of a given conductance is proportional to the width of the stable region. The agreement of the calculated histogram with the experimental one is similar to the correspondence shown in our Fig. 1: Outside the first two beat regions the calculated peaks agree quite well with the experimental ones but in the beat regions the theory shows two peaks with low intensity. Kassubek et al. did not discuss the attenuation of the conductance peaks as a supershell effect.

In the semiclassical model described above the closed orbits inside a circle are denoted by $(M, Q)$, where $M$ is the number of vertices and $Q$ is the winding number. The labels $(2,1),(3,1),(4,1)$, etc., denote the pendulum, triangle, square, etc., orbits, respectively. The labels $(5,2),(7,2)$, etc., correspond to the star orbits with 5,7 , etc., vertices, respectively, and $(4,2),(6,2)$, etc., are the second harmonics of the main band orbits $(2,1),(3,1)$, etc. In order to analyze the shell and supershell structures seen in the surface energy (Fig. 1) in terms of the classical orbits we have Fourier transformed the oscillating part $2 \pi R\left(\sigma-\sigma_{\mathrm{LDM}}\right)$. The modulus of the result is given in Fig. 2. The frequencies of the different orbits inside a circle are given by ${ }^{6}$

$$
f(M, Q)=M \sin (Q \pi / M) / \pi .
$$

In Fig. 2 the tips of the arrows denote frequencies $f(M, Q)$. The dominating peaks correspond to the pendulum and triangle orbits. The interference of these two frequencies gives the main features of the beat structure as discussed by Yanson et al. ${ }^{5}$ The two frequencies are well separated giving a relatively short distance between the adjacent beats on the $K_{F} R$ axis. In contrast to the spherical symmetry, the pendu- lum orbit has much importance because the triangle, square, etc., orbits now have a degeneracy similar to that of the pendulum orbit. Our analysis indicates that the importance of the square orbit is much smaller than that of the pendulum and triangle orbits. In contrast, the peak corresponding to the five-point star orbit is surprisingly clear. Also the peak of the seven-point star orbit is clearly distinguished.

Yanson et al. ${ }^{5}$ have Fourier transformed their conductance histogram. The peak intensities cannot directly be compared with our data in Fig. 2 arising from energy oscillations. Moreover, the experimental result may depend on kinetic aspects, such as the diffusion of atoms, so that the experimental results are not directly related to the equilibrium energetics of the wires. However, the experimental Fourier transform of the conductance histogram shows a clear peak corresponding to the pendulum orbit, and the triangle and square orbits give rise to a single peak with a higher intensity. It is also interesting to note that in the experimental data by Yanson $e t a l .{ }^{5}$ there are two peaks close to the frequencies of $1.5\left(K_{F} R\right)^{-1}$ and $1.7\left(K_{F} R\right)^{-1}$ corresponding to the five- and and seven-point star orbits.

Yanson et al. ${ }^{5}$ calculated the oscillating part of the thermodynamic potential using a parabolic shape for a hard-wall potential and a semiclassical expansion. Their Fourier transform gives similar intensity ratios between the pendulum, triangle, and square orbits as our data. Yanson et al. omitted the $Q \geqslant 2$ terms and the comparison with experiments at frequencies $\geqslant 1 / K_{F} R$ was not possible. Kassubek et al. ${ }^{17}$ Fourier transformed their simulated conductance histogram. In their plot, the intensity of the pendulum orbit is smaller than that of the triangle orbit, in agreement with experiment, but they do not show the high-frequency part $\left(\geqslant 1.2 / K_{F} R\right)$.

The shell and supershell structures observed in nanowires are due to occupied electron states near the Fermi energy. There is a correspondence between the semiclassical model and the quantum-mechanical level structure. ${ }^{18}$ In an energy level structure diagram for the cylindrical symmetry the classical orbits $(M, Q)$ are observed by the help of the quantum numbers $m$ and $n$ of the confined transversal motion. The classical orbit corresponds to quantum number differences between two nearly degenerate and successive levels so that $(M, Q)=(\Delta m, \Delta n)$. In the present case we consider the bottoms of occupied subbands near the Fermi level. The shell and supershell structures can be seen as oscillations of physical quantities as a function of the system size. For a system of fixed size they can be seen in the distribution of the electron levels as a function of the energy.

Figure 3 gives the bottoms of subbands for the widest wire we have calculated, i.e., $K_{F} R=22.78$. The angular momenta $m$ of the levels increase to the right and the $n$ quantum numbers upwards so that the levels with the same $n$ are connected with thin curves. Starting from the bottom of the figure, the levels $[m, n]=[0,2]$ and $[2,1]$ become degenerate first. This means the occurrence of the pendulum orbit $(M, Q)=(2,1)$ and a completed shell for a wire of about $K_{F} R=4.5$ (compare Fig. 1). Thereafter the degenerations are lost within the lowest shadowed region. The region corresponds to the first quantum beat at about $K_{F} R=6$ and the level structure indicates that the pendulum orbit $(2,1)$, of 


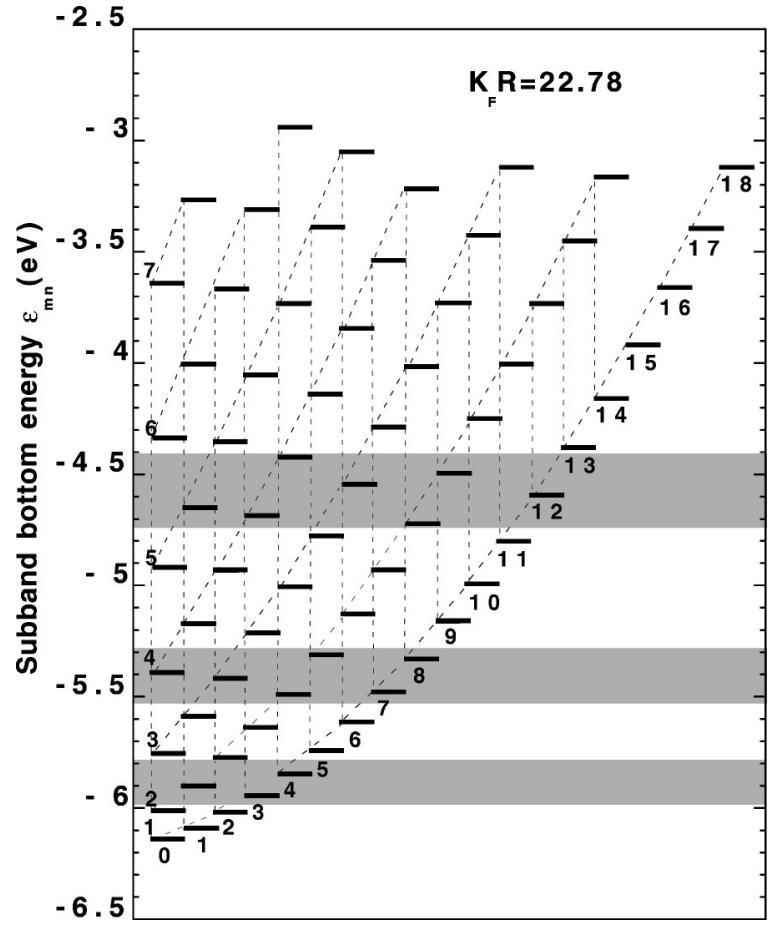

FIG. 3. Bottoms of subbands for the Na stabilized-jellium wire with $K_{F} R=22.78$. The angular momenta $m$ are given by the numbers below lines corresponding to $n=1$. The radial quantum numbers $n$ are given by the numbers above the $m=0$ lines. The levels with the same $n$ values are connected with dashed paraboliclike curves. The shadowed bands correspond to the regions of quantum beats.

levels $[1,2]$ and $[3,1]$, competes with the triangle orbit $(3,1)$ corresponding to $[1,2]$ and $[4,1]$. Above the first beat region five-star orbits $(5,2)$ appear: first formed by the levels $[0,3]$ and $[5,1]$ and then by $[1,3]$ and $[6,1]$. Two shell completions take place. The occurrence of the five-star orbits explains the strength of the corresponding peak in the Fourier transform in Fig. 2. The second shadowed band in Fig. 3 gives the region of the second beat $\left(K_{F} R=10.5\right)$. The degenerations corresponding to the pendulum and triangle orbits remain for three pairs of levels, but in the middle the structure of levels $[2,3],[4,2]$, and $[5,2]$ indicates the destructive interference between the pendulum and triangle orbits. The interpretation agrees with the supershell period corresponding to these orbits. The period is inversely proportional to their frequency difference, i.e., on the $K_{F} R$ scale it is about 5 units.

Above the second beat the bottoms of subbands again line up better. Degenerations corresponding to higher-order orbits such as $(5,2)$ and $(6,2)$ occur. Inside the third beat band, structures corresponding to the competition between the pendulum and triangle orbits occur again. Above that the occurrence of larger $m$ values gradually washes the shell and supershell structures. It is interesting to note that a degeneracy corresponding to the square orbit $(4,1)$ does not occur before the highest levels of our calculation, i.e., the levels [14,2] and $[18,1]$ are nearly degenerate. This means that the square orbit is not important for narrow wires in accordance with the Fourier transform (Fig. 2). The minor importance of the square orbit is a consequence of the rather soft increase of the potential near the surface of the wire. ${ }^{18}$

In conclusion, we have studied the stability of sodium quantum wires using a uniform cylindrical model and solving for the quantum-mechanical electron structures selfconsistently. The total energy per unit length shows shell and supershell structures in a quantitative agreement with $\mathrm{MCB}$ experiments. The quantum-mechanical electronic structures of the stabilized-jellium cylinders are analyzed in terms of a semiclassical picture, discussing the relative importance of the different classical electron orbits within the circular perpendicular cross section of the wire. The shell and supershell structures are shown to be dominated by the classical pendulum and triangle orbits. The five-star orbit is shown to give an important contribution at higher frequencies. These findings reinforce the previous semiclassical analysis and extend them to higher frequencies.

One of the authors (E.O.) acknowledges the Spanish Ministerio de Educación y Cultura for support (PB98-0780$\mathrm{CO} 2$ ). This work has also been supported by the Academy of Finland through its Centre of Excellence Programme (2000-2005).
${ }^{1}$ Nanowires, edited by P.A. Serena and N. García, Vol. 340 of the Nato ASI Series E: Applied Sciences (Kluwer, Dordrecht, 1997).

${ }^{2}$ A.I. Yanson et al., Nature (London) 400, 144 (1999).

${ }^{3}$ J.A. Torres et al., Phys. Rev. B 49, 16581 (1994).

${ }^{4}$ W.A. de Heer, Rev. Mod. Phys. 65, 611 (1993); M. Brack, ibid. 65, 677 (1993)

${ }^{5}$ A.I. Yanson, I.K. Yanson, and J.M. van Ruitenbeek, Phys. Rev. Lett. 84, 5832 (2000).

${ }^{6}$ R. Balian and C. Bloch, Ann. Phys. (N.Y.) 69, 76 (1972).

${ }^{7}$ C. Yannouleas, E.N. Bogachek, and U. Landman, Phys. Rev. B 57, 4872 (1998).

${ }^{8}$ C. Höppler and W. Zwerger, Phys. Rev. B 59, R7849 (1999).
${ }^{9}$ R.O. Jones and O. Gunnarsson, Rev. Mod. Phys. 61, 689 (1989).

${ }^{10}$ N. Zabala et al., Phys. Rev. B 59, 12652 (1999).

${ }^{11}$ C. Yannouleas and U. Landman, J. Phys. Chem. B 101, 5780 (1997).

${ }^{12}$ P. Ziesche et al., J. Phys.: Condens. Matter 5, 9049 (1993).

${ }^{13}$ A. García-Martín, J.A. Torres, and J.J. Sáenz, Phys. Rev. B 54, 13 448 (1996).

${ }^{14}$ Yu.V. Sharvin, Zh. Éksp. Teor. Fiz. 48, 984 (1965) [Sov. Phys. JETP 21, 655 (1965)].

${ }^{15}$ R. Landauer, IBM J. Res. Dev. 1, 223 (1957).

${ }^{16}$ J. Bürki et al., Phys. Rev. B 60, 5000 (1999).

${ }^{17}$ F. Kassubek et al., Nonlinearity 14, 167 (2000).

${ }^{18}$ A. Bohr and B.R. Mottelson, Nuclear Structure, Vol. II (W. A. Benjamin, London, 1975). 